\title{
The Effects of 5-HTR1A Polymorphism on Cingulum Connectivity in Patients with Panic Disorder
}

\author{
Seung Taek Yu', Min-Kyoung Kim ${ }^{1 *}$, Borah Kim¹, Eunhye Yoo², Jun-Yeob Lee ${ }^{3}$, \\ Kang Soo Lee ${ }^{4}$, Ah Young Choe', Ki-Hwan Yook', Tai Kiu Choi', and Sang-Hyuk Lee ${ }^{1 凶}$ \\ 1'Department of Psychiatry, CHA Bundang Medical Center, CHA University, Seongnam, Republic of Korea \\ ${ }^{2}$ Department of Radiology, CHA Bundang Medical Center, CHA University, Seongnam, Republic of Korea \\ ${ }^{3}$ Department of Psychiatry, CHA Gumi Medical Center, CHA University, Gumi, Republic of Korea \\ ${ }^{4}$ Department of Psychiatry, CHA Gangnam Medical Center, CHA University, Seoul, Republic of Korea
}

Objective Serotonin-1A receptors (5-HTR1A) is suggested to be involved in the etiology of several psychiatric disorders including panic disorder (PD). A few imaging studies have suggested the alterations of the cingulum bundle in PD. The objective of this study is to examine the structural changes of cingulum related to the 5-HTR1A polymorphism rs6295 in the patients with PD.

Methods Thirty-two right-handed patients with PD [11 men, 21 women; $40.34 \pm 13.17$ (mean \pm SD) age] who met the diagnostic criteria in Structured Clinical Interview for DSM-IV were examined by means of MRI at 3 Tesla. We divided the patients with PD into CC genotype group and non CC genotype group (GG/CG genotype group) of the 5-HTR1A rs6295 polymorphism to compare the cingulum white matter connectivity.

Results Tract-based spatial statistics showed significantly increased fractional anisotropy (FA) values in cingulate gyrus process of left cingulum in 5-HTR1A CC genotype compared to GG/CG genotype in PD. Significant positive correlations were shown between the Albany Panic and Phobia Questionnaire (APPQ) interoceptive fear subscale scores, the Anxiety Sensitivity Inventory-Revised fear of publicly observable anxiety reaction subscale scores and FA values of cingulate gyrus process of left cingulum in 5-HTR1A rs6295 GG/CG genotype group. In CC genotype group, APPQ total, APPQ agoraphobia subscale and APPQ social phobia subscale scores also showed significant positive correlations with FA values of hippocampal process of right cingulum.

Conclusion This preliminary study suggests that 5-HTR1A polymorphism may be associated with the cingulum white matter connectivity in PD.

Psychiatry Investig 2013;10:399-406

Key Words Panic disorder, 5-HTR1A polymorphism, Cingulum, Connectivity, Neuroimaging.

\section{INTRODUCTION}

Panic disorder (PD) is characterized by recurrent unexpected panic attacks followed by persistent worry about additional attacks or about the consequences of the attacks, ${ }^{1}$ which is associated with functional impairment. ${ }^{2,3}$ Family and twin studies have proposed a genetic contribution to the pathogenesis

\section{Received: November 23, 2012 Revised: January 29, 2013}

Accepted: February 4, 2013 Available online: December 16, 2013

$\triangle$ Correspondence: Sang-Hyuk Lee, MD, PhD

Department of Psychiatry, CHA Bundang Medical Center, CHA University,

59 Yatap-ro, Bundang-gu, Seongnam 463-712, Republic of Korea

Tel: +82-31-780-5694, Fax: +82-31-780-5862

E-mail: leesanghyuk@yahoo.com

*These authors contributed equally to this work and should be considered cofirst authors.

(a) This is an Open Access article distributed under the terms of the Creative Commons Attribution Non-Commercial License (http://creativecommons.org/licenses/bync/3.0) which permits unrestricted non-commercial use, distribution, and reproduction in any medium, provided the original work is properly cited. of $\mathrm{PD}$, with an estimated heritability of up to $48 \%{ }^{4}$

Serotonin-1A receptor (5-HTR1A) plays an important role in the regulation of the brain serotonin system. ${ }^{5}$ A common C(-1019)G single nucleotide (rs6295) polymorphism in the promoter region of the 5-HT1A receptor gene is associated with the autoregulation of the serotonergic neurotransmission. ${ }^{6}$ The G(-1019) allele has been shown to enhance the 5-HT1A autoreceptor expression on raphe nucleus, and activation of the 5-HT1A receptors leads to the hyperpolarization and decrease in neuronal excitability of serotonin neurons, resulting in the inhibition of the neurotransmitter release.

Disturbances in 5-HTR1A functions might contribute to pathogenesis of mental illness, ${ }^{7,8}$ including PD. ${ }^{9}$ Recent studies of animal models have demonstrated that disturbances in 5-HTR1A functions would contribute to anxiety-like behaviors. ${ }^{10-12}$ However, the association studies of the 5-HTR1A rs6295 polymorphism with PD have reported mixed results. 
Several studies have insisted that the G(-1019) allele could be a predisposing factor for $\mathrm{PD}^{9,13}$ whereas others have suggested that there is no significant evidence of the allelic association of the G(-1019) in PD. ${ }^{14}$

Neuroimaging studies might help clarify the association of the 5-HTR1A polymorphism with PD compared to inconsistencies of genetic association studies. Fakra et al. ${ }^{15}$ reported that the G(-1019) allele is associated with relatively decreased amygdala reactivity and related trait anxiety. The 5-HTR1A polymorphism has been found to be associated with prefrontal cortex and amygdala activation induced by emotional stimuli in PD. ${ }^{16}$

Previously, the fear circuit of PD was proposed to consist of the hippocampus, amygdala, thalamus, hypothalamus, brainstem, prefrontal cortex, insula and cingulate, which interact with one another to moderate panic responses. ${ }^{17,18}$ The cingulum bundle, a major link within the limbic-cortical networks, originates within the white matter of the temporal pole and runs posterior and superior into the parietal lobe, then turns around the corpus callosum, into the frontal lobe, terminating anterior and inferior to the genu of the corpus callosum in the orbital-frontal cortex. ${ }^{19}$ Han et al. reported that patients with PD have increased cingulum white matter connectivity in the left anterior and right posterior cingulate regions. ${ }^{20}$

However, no study has examined the relationship between the 5-HTR1A polymorphism and the connectivity of the cingulum in PD. The objectives of the present study were to 1) test the hypothesis that the 5-HTR1A polymorphism influences the cingulum white matter connectivity of PD and 2) explore whether the relationship between the alteration of the cingulum regions and clinical severity of $\mathrm{PD}$ is influenced by the 5-HTR1A polymorphism.

\section{METHODS}

Thirty-two right-handed patients with PD [21 women and 11 men; age, $40.34 \pm 13.17$ (mean $\pm S D$ ) years] were investigated in this study. All subjects were 18 to 60 years old and of Korean descent. Subjects were recruited trhough advertisements in an outpatient clinic between January 2011 and July 2012.

Patients with PD who met the Diagnostic and Statistical Manual of Mental Disorders, 4th Edition, Text Revision (DSMIV-TR), criteria for PD with or without agoraphobia using the structured clinical interview to assess DSM-IV-TR (SCID) Axis I disorders were enrolled in this study. Only patients with primary PD were included; secondary lifetime diagnoses were major depression in four patients and generalized anxiety disorder in three patients. Exclusion criteria included any history of schizophrenia, alcohol and substance abuse or de- pendence, mental retardation, current or past serious medical or neurological disorders, contraindications to MR scanning including metal implants, and pregnancy. Prior to the commencement of the study, all patients began treatment with a selective serotonin re-uptake inhibitor including paroxetine, or escitalopram, and benzodiazepines as anxiolytics, including alprazolam or clonazepam within one week (mean $\pm S D$, $3.52 \pm 2.52$ days).

Patients with PD were assessed for the clinical severity of their anxiety symptoms using the self-report version of Panic Disorder Severity Scale (PDSS), ${ }^{21}$ the Albany Panic and Phobia Questionnaire (APPQ), ${ }^{22}$ and the Anxiety Sensitivity Inventory-Revised (ASI-R). ${ }^{23}$ APPQ was developed to assess fear of activities that might induce physical sensation in panic patients. It includes three subscales: agoraphobia, social phobia, and interoceptive fear. ASI-R, the most commonly used measure of anxiety sensitivity, consists of fear of respiratory symptoms, fear of publicly observable anxiety reactions, fear of cardiovascular symptoms, and fear of cognitive dyscontrol.

All study procedures complied with CHA Bundang Medical Center Institutional Review Board regulations, Declaration of Helsinki, and the principles of Good Clinical Practice. After a complete description of the study was given to the subjects, written informed consent was obtained.

Diffusion data were acquired on a 3 Tesla GE Signa HDxt scanner (GE Healthcare, Milwaukee, WI, USA). Diffusionweighted images were acquired using an echo planar imaging (EPI) sequence, with the following parameters: repetition time (TR) $17000 \mathrm{~ms}$, echo time (TE) $108 \mathrm{~ms}$, field of view (FOV) 24 $\mathrm{cm}, 144 \times 144$ matrix, $1.7 \mathrm{~mm}$ slice thickness, and voxel size $1.67 \times 1.67 \times 1.7 \mathrm{~mm}^{3}$. A double echo option was used to reduce eddy current-related distortions. To reduce the impact of EPI spatial distortions, an 8-channel coil and an array of spatial sensitivity encoding techniques (ASSET, GE Healthcare) with a sensitivity encoding (SENSE) speed-up factor of 2 were used. Seventy axial slices parallel to the anterior commissure-posterior commissure (AC-PC) line covering the whole brain were acquired in 51 directions with $b=900 \mathrm{~s} / \mathrm{mm}^{2}$. Eight baseline scans with $b=0 \mathrm{~s} / \mathrm{mm}^{2}$ were also acquired. Diffusion-Tensor Images (DTIs) were estimated from the diffusion-weighted images using the least-squares method (approximate scan time $=17$ minutes).

Voxel-wise statistical analysis of the fractional anisotropy data was performed using Tract-Based Spatial Statistics (TBSS) version 1.2, implemented in FMRIB Software Library (FSL version 4.1, Oxford, UK, http://www.fmrib.ox.ac.jk/fsl) according to the standard procedure described in detail. First, DTI preprocessing, including skull stripping using the Brain Extraction Tool (BET) and eddy current correction, were performed using the FSL. FA images were created by fitting a 
tensor model to the raw diffusion data. All subjects' FA data were then aligned into the standard space (Montreal Neurologic Institute 152 standard) using the FMRIB's Nonlinear Image Registration Tool (FNIRT). All transformed FA images were combined and applied to the original FA map, resulting in a standard-space version FA map. All transformed FA images were averaged to create a mean FA image, which was then thinned (skeletonized) to create a mean FA skeleton, taking only the centers of WM tracts. The skeleton was thresholded by FA $>0.2$ (TBSS default) to include only major fiber bundles. Mean FA skeletons were multiplied using the John Hopkins University (JHU) DTI-based probablistic tractography atlas, which contains cingulum bundle white matter tracts/regions. Then, the cingulum regions of interest (ROI) were extracted to perform voxel-wise statistic analysis.

Statistical analysis was performed voxel by voxel to detect regions of significant differences of FA among two groups by using nonparametric permutation tests with a correction for multiple comparisons using the FSL Randomise program. To achieve accurate inference, including full correction for multiple comparisons over space, we used permutation-based nonparametric inference within the framework of the general linear model tested with 5,000 permutations; the significance level was set to $\mathrm{p}<0.05$. Multiple comparisons were corrected with threshold-free cluster enhancement (TFCE), which allowed us to avoid making an arbitrary choice of the clusterforming threshold while preserving the sensitivity benefits of cluster-wise correction. To further reduce the possibility of false-positive results, only clusters with more than 50 contiguous voxels were considered in the analysis. To assess the correlation analysis, the cingulum DTI data were analyzed using the TBSS General Liner Model (GLM) regression analysis with PDSS, APPQ, ASI-R and each subscale as a factor.

To analyze the 5-HTR1A (rs6295) polymorphism, Genomic DNA was extracted from blood (stored frozen) using GDEXTM II Genomic DNA Extraction Kit (Intron Biotechnology, Seongnam, Korea) according to the manufacturer's protocol. The detection of SNP was based upon an analysis of the primer extension products generated from previously amplified genomic DNA using a chip-based MALDI-TOF mass spectrometry platform (Sequenom, San Diego, CA, USA). The general procedures were performed according to the manufacturer's standard protocol. The PCR reaction was performed in a volume of $5 \mu \mathrm{L}$ containing 1X PCR buffer (Solgent, Daejeon, Korea), $2.5 \mathrm{mM}$ of $\mathrm{MgCl}$, $0.2 \mathrm{mM}$ of each dNTP, $0.1 \mathrm{U}$ of hTaq Polymerase (Solgent), $200 \mathrm{nM}$ of each primer (5'-ACGTTGGATGGTCAGTCTCCCAATTATTGC and 5'-ACGTTGGATGCGAGAACGGAGGTAGCTTTT), and 4.0 ng of genomic DNA. The reaction consisted of denaturation at $95^{\circ} \mathrm{C}$ for $15 \mathrm{~min}$, followed by $45 \mathrm{cycles}$ of $95^{\circ} \mathrm{C}$ for $20 \mathrm{sec}, 56^{\circ} \mathrm{C}$ for $30 \mathrm{sec}$, and $72^{\circ} \mathrm{C}$ for $1 \mathrm{~min}$, with a final extension at $72^{\circ} \mathrm{C}$ for $3 \mathrm{~min}$. Following PCR, unincorporated dNTPs were removed by adding $0.3 \mathrm{U}$ of shrimp alkaline phosphatase (Sequenom) and incubating for $40 \mathrm{~min}$ at $37^{\circ} \mathrm{C}$, followed by 5 min at $85^{\circ} \mathrm{C}$ for enzyme inactivation. In the homogeneous MassEXTENDTM reaction, the total volume of each reaction was $9 \mu \mathrm{L}$, including thermosequenase (Sequenom), appropriate termination mix, and $5 \mathrm{uM}$ of extension primer (5'AAGACCGAGTGTGTCTTC). The primer extension protocol was started at $94^{\circ} \mathrm{C}$ for $2 \mathrm{~min}$, followed by 55 cycles of $94^{\circ} \mathrm{C}$ for $5 \mathrm{sec}, 52^{\circ} \mathrm{C}$ for $5 \mathrm{sec}$, and $72^{\circ} \mathrm{C}$ for $5 \mathrm{sec}$. After desalting the reaction product with SpectroCLEANTM (Sequenom), samples were dispensed on 384 well SpectroCHIPTM (Sequenom) using SpectroJETTM (Sequenom). The SpectroCHIPs were analyzed in the fully automated mode with the MALDI-TOF MassARRAY system (Sequenom). After overall automatic measurements, any assays that had bad peaks were checked again manually.

\section{RESULTS}

\section{Genotyping}

We divided the patients with PD into the $\mathrm{CC}$ genotype group and the non-CC genotype group (GG/CG genotype group) of the 5-HTR1A rs6295 polymorphism to evaluate the differences in cingulum white matter connectivity between the two groups. Genotype distributions of all 32 patients with PD were in accordance with the Hardy-Weinberg equilibri$\mathrm{um}\left(\chi^{2}=2.31, \mathrm{df}=1, \mathrm{p}=0.12\right)$. Subjects in the respective genotype groups were not significantly different in terms of age, gender, and the frequencies of generalized anxiety disorder and major depression. None of the scores of the rating scales were significantly different according to the 5-HTR1A rs6295 polymorphism (Table 1).

\section{Tract-based spatial statistics results}

Results of TBSS analysis between the CC genotype group and the GG/CG genotype group yielded one cluster of significant voxels on the white matter skeleton (corrected $\mathrm{p}<0.05$ ), which was located in the cingulate gyrus process of the left cingulum. An increased FA value was found in subjects with the CC genotype compared to subjects with the GG/CG genotype (Figure 1).

We conducted a correlational analysis between the clinical severity of anxiety symptoms and the FA values of the cingulum region for each CC and GG/CG genotype group. In the GG/CG genotype group, FA values of the cingulate gyrus process of the left cingulum showed significant positive correlations with the APPQ interoceptive fear subscale $(r=0.814$, $\mathrm{p}=0.008)$ and the ASI-R fear of publicly observable anxiety re- 
Table 1. Sociodemographic and clinical characteristics for panic disorder (PD) patients

\begin{tabular}{|c|c|c|c|c|}
\hline & $\mathrm{CC}(\mathrm{N}=21)$ & GG and $C G(N=11)$ & $\mathrm{t}$ & $\mathrm{p}$ \\
\hline Gender, Male/Female & $6 / 15$ & $5 / 6$ & & $0.44^{*}$ \\
\hline Age (years, mean $\pm S D$ ) & $40 \pm 11.97$ & $41 \pm 15.82$ & -0.184 & 0.856 \\
\hline Depression (number) & 3 & 1 & & $1^{*}$ \\
\hline Generalized anxiety disorder (number) & 2 & 1 & & $1^{*}$ \\
\hline PDSS score $($ mean \pm SD) & $10.43 \pm 5.33$ & $13.00 \pm 6.75$ & -1.016 & 0.329 \\
\hline APPQ score (mean $\pm S D)$ & $51.80 \pm 44.54$ & $41.11 \pm 40.43$ & 0.644 & 0.528 \\
\hline APPQ agoraphobia & $19.48 \pm 17.09$ & $15.44 \pm 19.19$ & 0.544 & 0.595 \\
\hline APPQ social phobia & $15.62 \pm 17.45$ & $16.44 \pm 16.47$ & -0.124 & 0.903 \\
\hline APPQ interoceptive fear & $16.71 \pm 14.39$ & $9.22 \pm 8.07$ & 1.812 & 0.082 \\
\hline ASI-R score (mean \pm SD) & $52.48 \pm 28.94$ & $52.75 \pm 29.52$ & -0.22 & 0.982 \\
\hline ASI-R fear of respiratory symptom & $20.67 \pm 10.91$ & $22.00 \pm 11.82$ & -0.277 & 0.786 \\
\hline ASI-R fear of publicly observable anxiety reaction & $10.67 \pm 7.88$ & $10.38 \pm 8.11$ & 0.087 & 0.932 \\
\hline ASI-R fear of cardiovascular symptom & $14.38 \pm 10.59$ & $14.63 \pm 8.91$ & -0.062 & 0.951 \\
\hline ASI-R fear of cognitive dyscontrol & $6.76 \pm 7.56$ & $6.22 \pm 6.92$ & 0.190 & 0.851 \\
\hline
\end{tabular}

CC/CG/GG, The genotypes of serotonin 1A receptor (5-HTR1A) gene C-1019G polymorphism (rs6295). *Fisher's exact test. SD: standard deviation, PDSS: Panic Disorder Severity Scale, APPQ: Albany Panic and Phobia Questionnaire, ASI-R: Anxiety Sensitivity Inventory-Revised
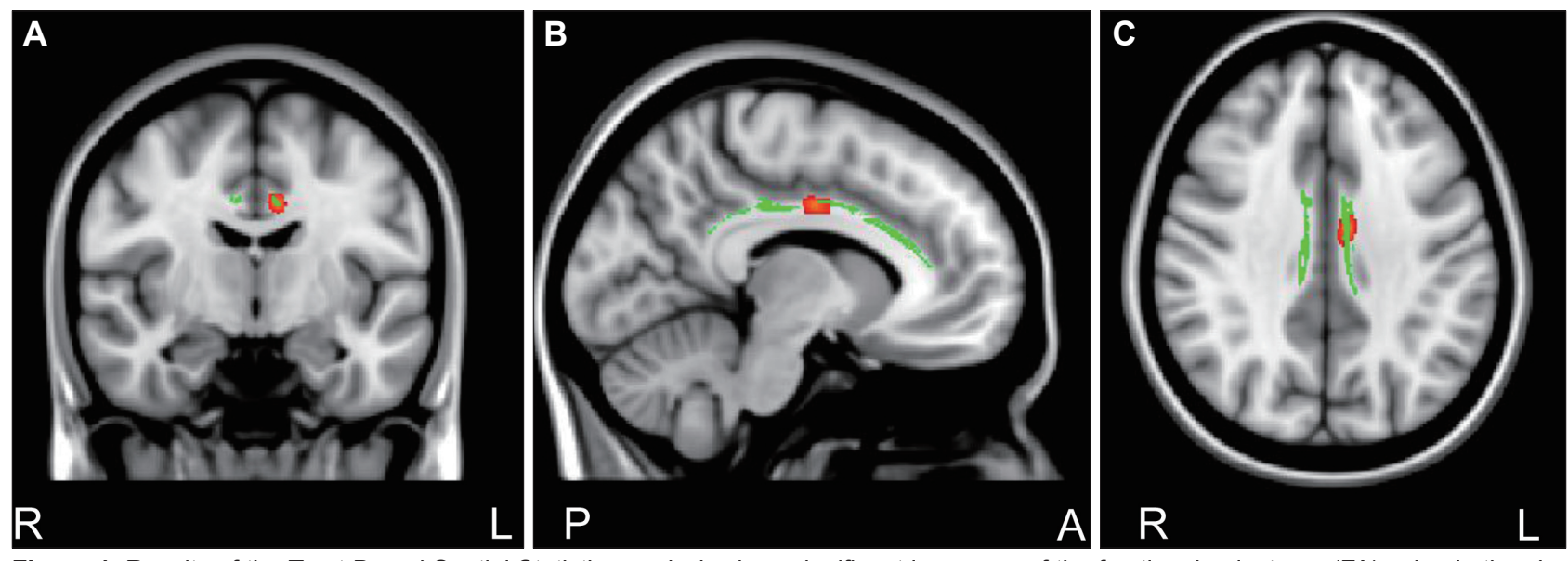

Figure 1. Results of the Tract-Based Spatial Statistics analysis show significant increases of the fractional anisotropy (FA) value in the cingulate gyrus process of the left cingulum in serotonin-1A receptors (5-HTR1A) rs6295 CC genotype group compared to the GG/CG genotype group in patients with panic disorder (PD). Voxels demonstrating significantly (corrected $p<0.05$ ) increased FA values for the CC genotype group compared to the GG/CG genotype group in patients with PD are shown in red-yellow. Voxels are thickened into local tracts and overlaid on the cingulum white matter skeleton (green). Images from coronal (A), sagittal (B), and transversal (C) views are shown for slices' coordinates: $x=98, y=120, z=107$.

action subscale scores $(\mathrm{r}=0.934, \mathrm{p}=0.001)$ (Table 2, Figure 2).

In the CC genotype group, the APPQ total, APPQ agoraphobia subscale, and APPQ social phobia subscale scores were shown to have significant positive correlations with FA values of the hippocampal process of the right cingulum (Table 2, Figure 3). FA values of the cingulate gyrus process of the left cingulum were not significantly correlated with the APPQ interoceptive fear subscale $(\mathrm{r}=-0.067, \mathrm{p}=0.773$ ) or ASI- $\mathrm{R}$ fear of publicly observable anxiety reaction subscale scores $(\mathrm{r}=-0.054$, $\mathrm{p}=0.816$ ).

The correlation coefficients $r$ values in the APPQ interoceptive fear subscale and ASI-R fear of publicly observable anxiety reaction subscale between the GG/CG and CC genotype groups were significantly different (APPQ interoceptive fear subscale: $\mathrm{p}=0.01$, ASI-R fear of publicly observable anxiety reaction subscale: $p<0.001$ ) when we analyzed the two $r$ values using the Fisher's $r$ to $z$ transformation (Figure 2).

After multiple comparison corrections [9 measurements (APPQ, ASI-R total and subscales $) \times 2$ groups $=18$; Bonferroni correction, level of significance alpha $=0.05 / 18 \fallingdotseq 0.003]$, the cingulate gyrus process of the left cingulum remained significant in the correlation analysis between the FA values and ASI-R fear of publicly observable anxiety reaction subscale scores. 
Table 2. Regions showing significant positive correlation between the anxiety-rating scale scores and fractional anisotropy values in 5-HTR1A rs6295 GG/CG genotype group and CC genotype group in patients with panic disorder

\begin{tabular}{clclc}
\hline $\begin{array}{c}\text { Genotype } \\
\text { group }\end{array}$ & \multicolumn{1}{c}{ Anxiety-rating scale } & $\begin{array}{c}\text { Peak coordinates* } \\
(\mathrm{X}, \mathrm{Y}, \mathrm{Z})^{\dagger}\end{array}$ & \multicolumn{1}{c}{ Anatomical locations } \\
\hline GG/CG & APPQ interoceptive fear & $96,109,108$ & Cingulate gyrus process of Cingulum, left & 0.008 \\
& ASI-R fear of publicly observable anxiety reaction & $98,111,107$ & Cingulate gyrus process of Cingulum, left & 0.001 \\
CC & APPQ & $72,90,66$ & Hippocampal process of Cingulum, right & 0.017 \\
& APPQ agoraphobia & $71,90,66$ & Hippocampal process of Cingulum, right & 0.029 \\
& APPQ social phobia & $72,90,66$ & Hippocampal process of Cingulum, right & 0.008 \\
\hline
\end{tabular}

${ }^{*}$ Talairach coordinates, ${ }^{\dagger}$ Foci for significant differences are listed (corrected $\left.\mathrm{p}<0.05\right)$. APPQ: Albany Panic and Phobia Questionnaire, ASI-R: Anxiety Sensitivity Inventory-Revised
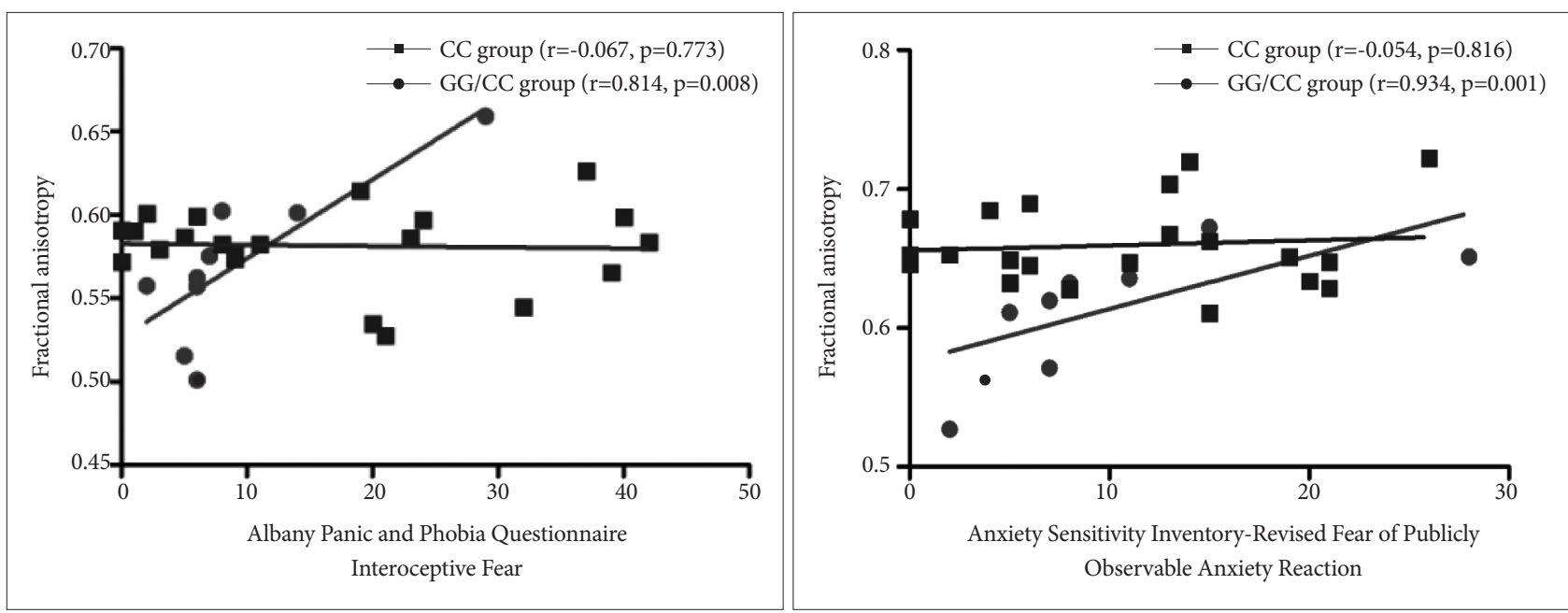

Figure 2. Significant positive correlations (corrected $p<0.05$ ) among the Albany Panic and Phobic Questionnaire $(A P P Q)$ interoceptive fear subscale scores, Anxiety Sensitivity Inventory-Revised (ASI-R) fear of publicly observable anxiety reaction subscale scores, and FA values of the cingulate gyrus process of the left cingulum in 5-HTR1A rs6295 GG/CG genotype group in patients with PD. Fisher's $r$ to $z$ transformation test showed a significantly different correlation of coefficient scores in APPQ interoceptive fear subscale scores and ASI-R fear of publicly observable anxiety reaction subscale scores between in 5-HTR1A rs6295 CC and GG/CG genotype groups.

\section{DISCUSSION}

To our knowledge, this is the first study to examine cingulum bundle white matter connectivity using diffusion tensor imaging in relation to the 5-HTR1A polymorphism in patients with PD. The present results showed significantly increased FA values in the cingulate gyrus process of the left cingulum in the 5-HTR1A CC genotype compared to the GG/ CG genotype in PD patients. A correlation analysis in the 5-HTR1A GG/CG genotype group showed a significant positive correlation among APPQ interoceptive fear, ASI-R fear of publicly observable anxiety reaction subscale scores, and FA values of the cingulate gyrus process of the left cingulum. In the CC genotype group, APPQ total, APPQ agoraphobia, and APPQ social phobia subscale scores were shown to be significantly positively correlated with the FA values of the hippocampal process of the right cingulum.

The 5-HT1A receptor gene transcription is modulated by a common C(-1019)G single nucleotide polymorphism in the promotor region of the 5-HT1A receptor gene. ${ }^{24,25}$ The C(-1019) allele binds the nuclear deformed epidermal autoregulatory factor-1-related protein to repress the 5-HT1A receptor gene transcription whereas the G(-1019) allele repeals repression by the protein, resulting in a higher expression of the 5-HT1A receptor. ${ }^{26-28}$ Because presynaptic 5-HT1A autoreceptors on the raphe nucleus play a major role in a negative autoregulatoty loop that inhibits the activity of the serotonergic neuron, we can assume that the G(-1019) allele enhances the 5-HT1A autoreceptor expression to reduce serotonergic neurotransmission. ${ }^{28-30}$ Previous studies have suggested that the G(-1019) allele is a risk allele that might be associated with aggression, ${ }^{31,32}$ suicidality, ${ }^{28,33,34}$ impulsivity, ${ }^{35}$ and several psychiatric disorders, including major depression ${ }^{28}$ and PD. ${ }^{36}$ However, the C(-1019) allele also showed increased frequency of anxiety disorders, and it is suggested that this might be due to increased amygdala activation. ${ }^{37}$ Combined with evidence of the association between the C(-1019) allele and ADHD, the 5HT1A-medicated inhibition of prefrontal and corticostriatal activity ight predispose individuals to various psychiatric disorders. ${ }^{38}$

Some reports have indicated that panic responses are influ- 

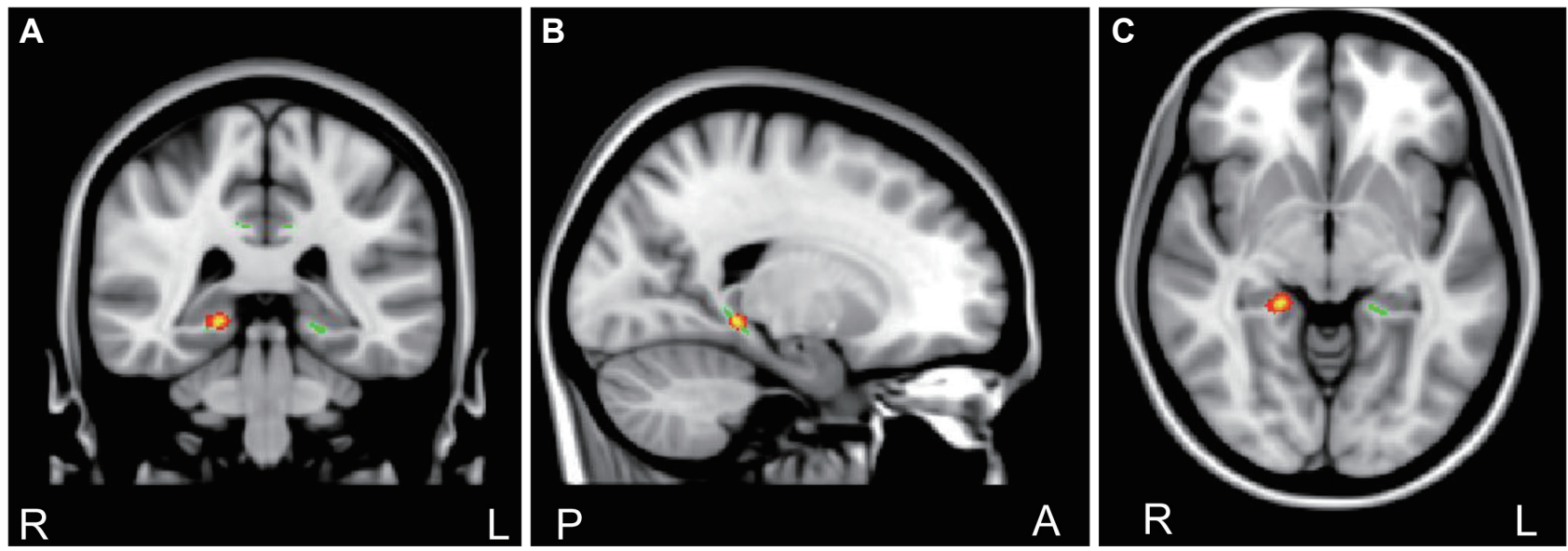

Figure 3. TBSS analysis showing clusters of voxels in the hippocampal process of the right cingulum with a significant positive correlation between APPQ scores and FA values in 5-HTR1A rs6295 CC genotype group in patients with PD. Voxels demonstrating significant positive correlation (corrected $p<0.05$ ) between the scores of APPQ and FA values of the white matter clusters in the CC genotype group in PD are shown in red-yellow. Voxels are thickened into local tracts and overlaid on the cingulum white matter skeleton (green). Images from coronal (A), sagittal (B), and transversal $(C)$ views are shown for the three slices' coordinates: $x=72, y=90, z=66(A), x=71, y=90, z=66(B), x=72$, $y=90, z=66(C)$.

enced by the 5-HTR1A polymorphism through the functional or structural change of the brain region. In patients with the G(-1019) allele, fearful stimuli were associated with a decreased activation of right prefrontal cortex regions and showed higher amygdala activation in response to happy faces. ${ }^{16}$ Fakra et al. ${ }^{15}$ reported that the 5-HTR1A rs6295 polymorphism is associated with amygdala reactivity and individual differences in trait anxiety. The present results showed significantly increased FA values among subjects with the 5-HTR1A CC genotype in the cingulate gyrus process of the left cingulum compared to subjects with the GG/CG genotype in PD. Therefore, the genotypes might influence functional or structural differences in PD or act as a moderator for the association between symptom severity and white matter connectivity.

Several brain-imaging studies have reported that the cingulum connectivity of the brain is associated with the regulation of mood and anxiety. ${ }^{39-42}$ In other words, it seems that cingulum connectivity is related to emotional regulation, memory and motor functions, visual-spatial memory functions, and fear response. ${ }^{43-45}$ One study showed that PD patients exhibit significantly greater FA values in left anterior and right posterior cingulate regions relative to healthy controls. ${ }^{20}$ Thus, combined with the current results, the 5-HTR1A polymorphism might be assumed to impact the cingulum white matter connectivity, which can regulate anxiety symptomatology.

Our result showed a significant positive correlation among APPQ interoceptive fear, ASI fear of publicly observable anxiety reaction subscale scores, and FA values of the cingulate gyrus process of the left cingulum in the GG/CG genotype group, whereas in the CC genotype group there were no such significant correlation. In addition, these correlation coefficients differed significantly between the GG/CG and CC gen- otype groups according to Fisher's z transformation analysis. Furthermore, unlike in the GG/CC group, APPQ total, APPQ agoraphobia subscale, and APPQ social phobia subscale scores were shown to have significant positive correlations with FA values of the hippocampal process of the right cingulum in the CC genotype group. These results suggest that the 5-HTR1A polymorphism can influence the cingulum connectivity, which might affect anxiety symptoms in PD.

The current preliminary study has several limitations. First, the sample size in each group was small. Although significantly increased FA in cingulum regions was found in the CC genotype compared to the GG/CG genotype in this study, further studies should include a larger number of patients for more accurate results. Second, we studied only patients with PD. To obtain a clearer understanding of the relationship between the 5-HTR1A polymorphism and the cingulum white matter connectivity of PD, a study should be conducted with a control group. In addition, the influence of medication and comorbidity with depression or generalized anxiety disorder on the cingulum white matter connectivity across genotype groups could not be ruled out completely. Third, although it was just few days of medication at scan and the primary diagnosis of all patients was PD, undetected factors other than the genotype itself might have contributed to the observed cingulum white matter connectivity changes in each 5-HTR1A genotype group.

In conclusion, the current study identified the altered cingulum white matter connectivity in patients with PD according to the genotype of the 5-HTR1A polymorphism. Altered white matter connectivity can be significantly correlated with symptom severity of PD. These findings suggest that the 5-HTR1A polymorphism might be associated with the cin- 
gulum white matter connectivity in $\mathrm{PD}$ patients.

\section{Acknowledgments}

This research was supported by the Basic Science Research Program through the National Research Foundation of Korea funded by the Ministry of Education, Science and Technology (2011-0023359) to S. H. Lee.

\section{REFERENCES}

1. American Psychiatric Association. Diagnostic and Statistical Manual of Mental Disorders, Text Revision (DSM-IV-TR). Washington, DC: American Psychiatric Association; 2000.

2. Hollifield M, Katon W, Skipper B, Chapman T, Ballenger JC, Mannuzza S, et al. Panic disorder and quality of life: variables predictive of functional impairment. Am J Psychiatry 1997;154:766-772.

3. Choi KW, Woo JM, Kim YR, Lee SH, Lee SY, Kim EJ, et al. Long-term escitalopram treatment in Korean patients with panic disorder: a prospective, naturalistic, open-label, multicenter trial. Clin Psychopharmacol Neurosci 2012;10:44-48.

4. Hettema JM, Neale MC, Kendler KS. A review and meta-analysis of the genetic epidemiology of anxiety disorders. Am J Psychiatry 2001; 158:1568-1578.

5. Lucki I. The spectrum of behaviors influenced by serotonin. Biol Psychiatry 1998;44:151-162.

6. Wu S, Comings DE. A common C-1018G polymorphism in the human 5-HT1A receptor gene. Psychiatr Genet 1999;9:105-106.

7. Le Francois B, Czesak M, Steubl D, Albert PR. Transcriptional regulation at a HTR1A polymorphism associated with mental illness. Neuropharmacology 2008;55:977-985.

8. Albert PR, Le Francois B, Millar AM. Transcriptional dysregulation of 5-HT1A autoreceptors in mental illness. Mol Brain 2011;4:21.

9. Neumeister A, Bain E, Nugent AC, Carson RE, Bonne O, Luckenbaugh DA, et al. Reduced serotonin type $1 \mathrm{~A}$ receptor binding in panic disorder. J Neurosci 2004;24:589-591.

10. Gross C, Zhuang X, Stark K, Ramboz S, Oosting R, Kirby L, et al. Serotonin1A receptor acts during development to establish normal anxiety-like behaviour in the adult. Nature 2002;416:396-400.

11. Heisler LK, Chu HM, Brennan TJ, Danao JA, Bajwa P, Parsons LH, et al. Elevated anxiety and antidepressant-like responses in serotonin 5-HT1A receptor mutant mice. Proc Natl Acad Sci U S A 1998;95:1504915054.

12. Parks CL, Robinson PS, Sibille E, Shenk T, Toth M. Increased anxiety of mice lacking the serotonin1A receptor. Proc Natl Acad Sci U S A 1998;95:10734-10739.

13. Choi WS, Lee BH, Yang JC, Kim YK. Association study between 5-HT1A Receptor Gene C(-1019)G Polymorphism and panic disorder in a Korean population. Psychiatry Investig 2010;7:141-146.

14. Rothe C, Gutknecht L, Freitag C, Tauber R, Mossner R, Franke P, et al. Association of a functional 1019C $>$ G 5-HT1A receptor gene polymorphism with panic disorder with agoraphobia. Int J Neuropsychopharmacol 2004;7:189-192.

15. Fakra E, Hyde LW, Gorka A, Fisher PM, Munoz KE, Kimak M, et al. Effects of HTR1A C(-1019)G on amygdala reactivity and trait anxiety. Arch Gen Psychiatry 2009;66:33-40.

16. Domschke K, Braun M, Ohrmann P, Suslow T, Kugel H, Bauer J, et al. Association of the functional -1019C/G 5-HT1A polymorphism with prefrontal cortex and amygdala activation measured with $3 \mathrm{~T}$ fMRI in panic disorder. Int J Neuropsychopharmacol 2006;9:349-355.

17. Gorman JM, Fyer MR, Goetz R, Askanazi J, Liebowitz MR, Fyer AJ, et al. Ventilatory physiology of patients with panic disorder. Arch Gen Psychiatry 1988;45:31-39.

18. Gorman JM, Kent JM, Sullivan GM, Coplan JD. Neuroanatomical hypothesis of panic disorder, revised. Am J Psychiatry 2000;157:493-505.

19. Schmahmann J, Pandya, D. Fiber Pathways of the Brain. New York:
Oxford University Press; 2006.

20. Han DH, Renshaw PF, Dager SR, Chung A, Hwang J, Daniels MA, et al. Altered cingulate white matter connectivity in panic disorder patients. J Psychiatr Res 2008;42:399-407.

21. Lim YJ, Yu BH, Kim JH. Korean panic disorder severity scale: construct validity by confirmatory factor analysis. Depress Anxiety 2007;24:95102.

22. Brown TA, White KS, Barlow DH. A psychometric reanalysis of the Albany Panic and Phobia Questionnaire. Behav Res Ther 2005;43:337355.

23. Lim YJ, Yu BH, Kim JH. Korean Anxiety Sensitivity Index-Revised: its factor structure, reliability, and validity in clinical and nonclinical samples. Depress Anxiety 2007;24:331-341.

24. Albert PR, Lembo P, Storring JM, Charest A, Saucier C. The 5-HT1A receptor: signaling, desensitization, and gene transcription. Neuropsychopharmacology 1996;14:19-25.

25. Albert PR, Lemonde S. 5-HT1A receptors, gene repression, and depression: Guilt by association. Neuroscientist 2004;10:575-593.

26. Gross CT, McGinnis W. DEAF-1, a novel protein that binds an essential region in a Deformed response element. EMBO J 1996;15:1961-1970.

27. Bottomley MJ, Collard MW, Huggenvik JI, Liu Z, Gibson TJ, Sattler M. The SAND domain structure defines a novel DNA-binding fold in transcriptional regulation. Nat Struct Biol 2001;8:626-633.

28. Lemonde S, Turecki G, Bakish D, Du L, Hrdina PD, Bown CD, et al. Impaired repression at a 5 -hydroxytryptamine $1 \mathrm{~A}$ receptor gene polymorphism associated with major depression and suicide. J Neurosci 2003;23:8788-8799.

29. Blier P, de Montigny C. Serotonin and drug-induced therapeutic responses in major depression, obsessive-compulsive and panic disorders. Neuropsychopharmacology 1999;21(2 Suppl):91S-98S.

30. Kennett GA, Marcou M, Dourish CT, Curzon G. Single administration of 5-HT1A agonists decreases 5-HT1A presynaptic, but not postsynaptic receptor-mediated responses: Relationship to antidepressantlike action. Eur J Pharmacol 1987;138:53-60.

31. Cleare AJ, Bond AJ. Ipsapirone challenge in aggressive men shows an inverse correlation between 5-HT1A receptor function and aggression. Psychopharmacology (Berl) 2000;148:344-349.

32. Parsey RV, Oquendo MA, Simpson NR, Ogden RT, Van Heertum R, Arango V, et al. Effects of sex, age, and aggressive traits in man on brain serotonin 5-HT1A receptor binding potential measured by PET using [C-11]WAY-100635. Brain Res 2002;954:173-182.

33. Boldrini M, Underwood MD, Mann JJ, Arango V. Serotonin-1A autoreceptor binding in the dorsal raphe nucleus of depressed suicides. J Psychiatr Res 2008;42:433-442.

34. Serretti A, Calati R, Giegling I, Hartmann AM, Moller HJ, Rujescu D. Serotonin receptor HTR1A and HTR2C variants and personality traits in suicide attempters and controls. J Psychiatr Res 2009;43:519-525.

35. Benko A, Lazary J, Molnar E, Gonda X, Tothfalusi L, Pap D, et al. Significant association between the C(-1019)G functional polymorphism of the HTR1A gene and impulsivity. Am J Med Genet B Neuropsychiatr Genet 2010;153B:592-599.

36. Strobel A, Gutknecht L, Rothe C, Reif A, Mossner R, Zeng Y, et al. Allelic variation in 5-HT1A receptor expression is associated with anxietyand depression-related personality traits. J Neural Transm 2003;110: 1445-1453.

37. Schenkel LC, Bragatti JA, Becker JA, Torres CM, Martin KC, de Souza $\mathrm{AC}$, et al. Serotonin gene polymorphisms and psychiatry comorbidities in temporal lobe epilepsy. Epilepsy Res 2012;99:260-266.

38. Tsuchida R, Kubo M, Kuroda M, Shibasaki Y, Shintani N, Abe M, et al. An antihyperkinetic action by the serotonin 1A-receptor agonist osemozotan co-administered with psychostimulants or the non-stimulant atomoxetine in mice. J Pharmacol Sci 2009;109:396-402.

39. Ebert D, Feistel H, Barocka A, Kaschka W. Increased limbic blood flow and total sleep deprivation in major depression with melancholia. Psychiatry Res 1994;55:101-109. 
40. Maddock RJ, Garrett AS, Buonocore MH. Posterior cingulate cortex activation by emotional words: fMRI evidence from a valence decision task. Hum Brain Mapp 2003;18:30-41.

41. Larish R, Klimke A, Vosberg H, Gaebel W, Mueller-Gaertner HW. Cingulate function in depression. Neuroreport 1997;8:i-ii.

42. Pillay SS, Gruber SA, Rogowska J, Simpson N, Yurgelun-Todd DA. fMRI of fearful facial affect recognition in panic disorder: the cingulate gyrus-amygdala connection. J Affect Disord 2006;94:173-181.
43. Devinsky O, Morrell MJ, Vogt BA. Contributions of anterior cingulate cortex to behaviour. Brain 1995;118:279-306.

44. Mezzasalma MA, Valenca AM, Lopes FL, Nascimento I, Zin WA, Nardi AE. Neuroanatomy of panic disorder. Rev Bras Psiquiatr 2004;26: 202-206.

45. Nobre AC, Sebestyen GN, Gitelman DR, Mesulam MM, Frackowiak RS, Frith CD. Functional localization of the system for visuospatial attention using positron emission tomography. Brain 1997;120:515-533. 